\title{
Sozialraumarbeit und die Stadt der Zukunft
} Auf dem Weg zur lernenden Gemeinde

Konrad Hummel

Dr. Konrad Hummel ist Beauftragter für die Konversion bislang militärisch genutzter Flächen in Mannheim. Internet http://www.mannheim.de/stadtgestalten/beauftragter-desoberbuergermeisters-konversion
Die Sozialraumarbeit muss auch Institutionen verändern, denn die Entwicklung neuer Nachbarschaften verändert die Spielregeln für alle Beteiligten.

In vormodernen Gesellschaften war das Verhältnis zwischen Alt und Jung geprägt vom Vorsprung des Wissens und der Verpflichtung der Weitergabe von Traditionen und Fürsorge der Alten für die Jungen. Die industrielle Moderne hat dieses Verhältnis fast umgedreht. Die erwerbstätige Generation hat für die Älteren zu sorgen, die Renten zu sichern. Gleichzeitig ist eine Kind- und Ausbildungszeit von erheblichem Zeit- und Finanzaufwand entstanden und beide Lebensenden, Jugend und Alter, gelten als »Kostenfaktoren ", als autonome Lebensphasen, die sich vielen Logiken der Arbeitswelt entziehen.

In nachindustriellen Wissensgesellschaften hat sich die Perspektive erneut verschoben. Es sind die Jüngsten im Bildungs- und Erwerbsprozess, deren Wissen und Kreativität vorangeht, dominiert und von denen Ältere nun lernen müssen. Gemeinsam haben die Generationen an Lösungen zu arbeiten, für die keine Generation einen natürlichen Wissensvorsprung hat. Gleichzeitig haben sich die Familienformen verändert. Sie haben sich vor allem so in die biografische Länge gezogen, dass Hochbetagte einer dritten und vierten Generation alleine und an anderen Orten als die Jungfamilien wohnen.

Alle Projekte einer Nach-Altenhilfe-Ära (Seniorenbüro, Mehrgenerationenhäuser) versuchen eine Art »Sozialraumstruktur « $\mathrm{zu}$ schaffen und Knoten- und Anlaufpunkte der Privaten im öffentlichen, gemeinsamen Raum zu sein.

Was hier altersspezifisch entstanden ist, hat sich auch in anderen gesellschaftlichen Veränderungen "abgebildet «, drei Beispiele:

- Gegen anonyme, großstädtische Entwicklungen entstanden Nachbarschaftszentren, Bürgerhäuser und Treffpunkte.
- Gegen vereinzelte Gesundheitsstrategien entstanden Selbsthilfekontaktstellen und Mütterzentren.

- Gegen die Auflösungstendenzen traditioneller Vereine und die Ohnmachtsgefühle engagementwilliger Einzelbürger entstanden Freiwilligenagenturen. Diese Einrichtungen sind Brücken zwischen privaten Lebensräumen und der Gesellschaft in Form ihrer Institutionen und Herrschaftsstrukturen. Sie sind im Zwischenraum von Wohnort und den zentralen Orten wie Rathaus, Betrieb, Schule etc. angesiedelt und schaffen mit ihren Kontaktnetzen selbst neue Räume. In der Regel aber benutzen Menschen solche Angebote, wollen aber nicht vollkommen von ihnen vertreten oder verwaltet werden. Sie ergänzen eine innere Landschaft, die, neben dem Sozialraum, der seinerseits sowohl aus territorialer Nachbarschaft als auch der Bekanntschaft sinnesgleicher Bürger besteht, geprägt ist von Familie, Freunden, Staatsbürgerschaft, Betriebs- und/oder Religionszugehörigkeit. Sozialräumliche Angebote sollten deshalb weniger fürsorglich oder anwaltschaftlich auftreten, sondern an einem solchen sozialpolitischen »Patchwork « der Betroffenen mithelfen.

\section{Sozialräume und Milieus}

Einige Quartiere können 2.000, andere 60.000 Bewohnerinnen und Bewohner haben. Da mögen lange Definitionen herangezogen oder der Begriff »sinnlich und historisch gewachsener Räume« strapaziert werden, um völlig unterschiedliche Quartiere zu begründen; nur hilft dies unter modernen Gesellschaftsbedingungen nicht weiter.

Nachbarschaften haben in einer postmodernen Welt eine doppelte Bedeutung - sie sind die umbaute Quartiersumgebung und sie sind das ähnlich denkende und handelnde Umfeld, die Gemeinschaft 
(Community), die so »tickt« wie ich. Nachbarn sind einerseits topografisch Wohnbezirks-, Quartiers-, Kiez oder Grätzlnachbarn und andererseits topologisch, wie in der Mathematik, Zugehörige gleicher Ordnung und Struktur ("geistige Nachbarn «). Es sind die Räume, in denen sich gruppenbezogene Lebensstile formieren, äußern und in einer Art Milieuperformanz in Kontakt treten. Wo Raumgrenzen im urbanen Raum diffus bleiben, werden ein Quartiers- und Milieubewusstsein und damit ein Quellelement von Bürgerengagement erschwert.

Die Neigung von Trägern, beispielsweise ihre Schulsozialarbeiter trägerspezifisch zusammenzuführen, sollte in sozialräumliche Kooperationen umgewandelt werden: Dann wären diese näher am Engagementumfeld. Der Wunsch ist groß, in überschaubare Räume zu gehen. Bürgerengagement und Stadtumbau, demografischer Wandel und Stadtteilbudgets, bürgernahe Sozialpolitik und Mobilisierung der Mieternachbarschaft fokussieren nahezu alle Dimensionen an einem konkreten, vermeintlich fußläufigen, überschaubaren Ort.

Moderne Dienstleistungstechniken erlauben die Illusion, diese Dimensionen bündeln zu können: Informationen, Wohnen, Arbeiten - alles so nahe wie möglich. Auch die Integration von $\mathrm{Mi}$ granten scheint nachbarschaftlich leichter zu erfolgen als auf rechtlich-normativ-abstrakter Ebene. Der Bedarf haushaltsbezogener Einfachhilfen im Alter mag in aktiver Nachbarschaft leichter zu befriedigen sein und der Wunsch nach Verbleib in der eigenen Wohnung legt den Quartiersansatz nahe.

Milieus sind unmittelbare Schnittstellen zum Bürgerengagement. Die Vergewisserung eines eigenen Lebensstils braucht den Weg über öffentliches Handeln, Konsumieren, Wohnen etc. Sie braucht eine Bühne und Performanz auch jenseits der Schul- oder Arbeitswelt. Wichtige Teile des Bürgerengagements sind geradezu Schaufenster bestimmter Milieus. Im Umkehrschluss heißt dies für die Quartiersentwicklung, dass ein Verschwinden klarer ethnischer und traditioneller Milieupräferenzen auch die Formen des Bürgerengagements in solchen Stadtteilen »uneindeutiger « macht, individueller, zersplitterter. Es entstehen dort, wo wir »früher « von Vielfalt, Durchmischung oder Nebeneinander im Quartier gespro- chen haben, Schnittstellen der verschiedenen Dimensionen von Nachbarschaft.

Im Bild gesprochen: Die Sozialräume sind wie geografische Einheiten an der Erdoberfläche, unter denen die Milieus als »tektonische Kontinentalplatten « schieben und wandern. Wo sie zu viel Spannung an ihren Schnittstellen aufbauen, brechen sie auf oder schieben sich nach oben oder unten weg - mit der Folge, dass sich Sozialräume neu formieren. Der emanzipative Gehalt des Sozialraumansatzes besteht darin, alle Milieus zu berücksichtigen, also nicht nur auf vordergründige Effekte und lautstarke Gruppen zu setzen.

\section{Nachbarschaftsarbeit und Stadtentwicklung}

Es geht in moderner hilfreicher Nachbarschaftsarbeit darum, Menschen und Gruppen in der Stadt zu unterstützen, damit sie selbst an den Schnittstellen ihrer eigenen Lebensmilieus und Differenzen $\mathrm{zu}$ anderen aktiv, prosozial und handlungsfähig werden. Kleine Alltagssorgen im Quartier müssen kein Gegensatz zu den großen Strategiefragen sein - entscheidend sind die Handlungschance und selbstbestimmte Handlungsfähigkeit im öffentlichen Raum. Unbearbeitete Differenzen und unterschiedslose Dienstleistungen können Handlungschancen eher blockieren.

Bürgerengagement lebt aus erlebten, gestaltbaren Differenzen und dem produktiven Versuch, den Anteil an Eigensinn und Individualität darin zu erkennen, das Gemeinsame in aktiven Grenzverletzungen, Aktionen und vielfältigen Kooperationen auszuloten. Je weniger wirksam große Familien und Institutionen uns ihre Normen vorgeben, desto mehr hat jeder sein Alter, sein Alleinsein, seine Religionszugehörigkeit, sein Hobby, seine Neigungen auf der Bühne der Gesellschaft selbst zu verteidigen.

In der Regel geschieht dies mit Brauchtumspflege der neuen Art: Frauen, Senioren oder Mitglieder bestimmter Glaubensrichtungen tun sich verstärkt zusammen. Es kommt darauf an, in der Öffnung dieser Milieus die allseitige Wertschätzung zu erhöhen. Seniorenclubs kochen für Wärmestuben, Motorradfahrervereine gestalten eine Behindertenausfahrt, Umweltgruppen kooperieren mit Managern. Differenzerfahrungen werden zu Reibungspunkten bei jenen Bürgern führen, die an ihrer "Sozialordnung « hängen. Selbst die Institutionen im Quartier tun sich schwer mit »Zuständigkeitsüberschreitungen « und haben zahlreiche Rechtfertigungen dafür: Die Fördervorschriften ihrer Zuschüsse erlaubten es nicht, die Professionen seien dazu nicht bestimmt, die Haftungsfragen seien ungeklärt und die eigene Klientel werde »überfremdet «.

Stadtentwicklung wird sich noch weiter weg von der Bauerschließung und Wohnbebauung hin zu einer stadtgesellschaftlichen Entwicklungsarbeit bewegen. Bürgerengagement und Bürgerbeteiligung dürfen in der Stadtentwicklung kein Mittel zum Zweck der Baurealisierung sein, sondern sie sind selbst ein Stück des Ziels, dem die Stadtentwicklung zu dienen hat: Menschen Raum zu bieten, in dem sie sich engagieren wollen, und Chancen und Anlässe für ein solches Engagement zu schaffen.

Es gilt, Quartiere so zu definieren, dass sie in Abwägung ökonomischer und zivilgesellschaftlicher Sachzwänge einen Aufforderungscharakter haben und einen Handlungsspielraum für Menschen bieten. Das gilt unter den Bedingungen des demografischen Wandels im Besonderen und für Milieus im Allgemeinen. Diese liegen »unter Tage « und müssen in ihrer Verschiedenheit "gehoben « werden. Sie schieben Generationen, Geschlechter, Religionen, Einkommensschichten und Ethnien zusammen und auseinander. An ihren Schnittstellen kann das Bürgerengagement erwachsen, das die Stadtgesellschaft letztlich zusammenhält. Sozialräumliche Arbeit fasst diesen räumlichen und sozialen Aspekt der Nachbarschaft zusammen.

\section{Doppelauftrag und Autonomie in helfender Nachbarschaft}

Sozialraumorientierte »Nachbarschaftsarbeit « rückt den doppelten Auftrag der sozialen und räumlichen Arbeit in den Mittelpunkt. Sie will die Verkürzung des Anspruchs durch Aufteilung der sozialen Arbeit in eine räumlich-funktionale, dienstleistungsorientierte Erbringung von Daseinsvorsorge und eine politisch-sozialnormative, engagementfördernde Animation und Organisierung von vordergrün- 
digen Interessen vermeiden, indem sie beides vor Ort macht: Kindergarten und Eltern mobilisieren.

In diesem Dilemma sind Wohlfahrtsverbände, Ämter, Bürgerinitiativen und Parteien verfangen und vermögen sich nicht oder nur unter Auflegung neuer Modellprogramme herauszulösen: Dienste aufbauen oder Menschen aktivieren!

In diesem Dilemma stecken offenkundig die Bildungseinrichtungen, weil sie es, wenn überhaupt, nur durch große Anstrengung fertigbekommen, den Nahraum zu »versorgen " - etwa als Ganztags-Grundschuleinrichtungen - oder durch Sonder-Modell-Privat-Campus Charakter die soziale Nachbarschaft, die Communities und Milieus gezielt zu erreichen, um den Preis kohäsiven $\mathrm{Zu}$ sammenhalts und lernfördernder Vielfalt.

Teile der Kindergarten- und Jugendamtsarbeit rutschen in diese Dynamik. Der Markt in der Altenpflege hat dies bei ambulanten Pflegediensten schon länger in Gang gesetzt. Allzu schnell wird dies mit Kostengründen entschuldigt. Die räumlichen Versorgungsformen werden wegen des Kostendrucks der öffentlichen Hand derart » nüchtern « und »karg « ausgestattet und selektiv (z. B. als Beratungsstelle kaum auf Migranten orientiert), dass der Druck auf die sozialen »Tendenzdienstleistungen « deutlich zunimmt. Diese versuchen ihrerseits fast hilflose Gegensteuerungen, indem sie Quoten und Stipendien schaffen, damit »andere « zustoßen können.

Diese Spaltung in Tausch- und Gebrauchswerte gilt nicht nur für die kaufkraftstärkeren Schichten und ihr Dienstleistungsprofil. Sie gilt auch zwischen Verwaltung und Zielgruppen im prekären Bereich.

\section{Eine besondere Herausforderung: die sozialräumliche Entwicklung ehemals militärisch genutzter Flächen}

Im Frühsommer 2010 wurde offiziell bekanntgegeben, dass ein vollständiger Abzug der US-Armee aus Mannheim und Heidelberg geplant ist. Besondere Brisanz erhält die Ankündigung auch durch den engen Zeitplan: bereits im Jahr 2015 soll der Abzug beendet sein - mit weitreichenden Folgen für die betroffenen Städte.

Allein in Mannheim werden innerhalb der kommenden Jahre insgesamt neun bisher militärisch genutzte Liegenschaften in einer Größenordnung von 510 Hektar frei, was etwa einem Siebtel des gesamten Stadtgebietes entspricht. Mit der Räumung dieser Flächen und Rückgabe an die Bundesrepublik Deutschland setzt ein Transformationsprozess ein, dessen Bewältigung die Stadt Mannheim vor große Herausforderungen stellt, gleichzeitig aber auch bedeutende Spielräume für neue - gerade auch qualitative - Entwicklungsziele für die Gesamtstadt eröffnet.

Quelle: http://www.konversion-in-mannheim.de nen? Die Kunden wollen anonym kaufen oder surfen sich in andere Wissenswelten. Sie erweitern ihren Raum und ihren Sozialraum um einen Marktraum. Diese Komplexität erscheint aus Sicht der Anbieter schwierig. Der Kunde kann sich entziehen. Für den endverbrauchenden Bürger ist die Marktsituation allerdings eine gewisse Entscheidungs-, Rückzugs- und Wechseloption. Er kann sich, neben die räumliche und seine soziale Nachbarschaft, eine virtuelle Nachbarschaft generieren.

In jedem Fall entstehen "Zwischenräume«. Eine der wenigen gemeindebezogenen Fallstudien zu Mittelstädten in Deutschland und ihrem Bürgerengagement beschreibt denn auch diese Situation als einen "Mix aus Gemeinsinn und Eigennutz « (Dörner/Vogt 2008, S. 211), eine längst überfällige Beschreibung der Verknüpfung scheinbar widerstrebender Werte im praktizierten Engagement (vgl. »Engagement-Viereck « bei Hummel 2010a, S. 95). Bürgerengagement hilft nicht nur im Zusammenhang mit dem Gemeinwohl und im Eigeninteresse, sondern generiert oft eigene soziale teilautonome Welten.

\section{Lernlandschaften und Nachbarschaft}

$\mathrm{Zu}$ den zentralen Herausforderungen im Verhältnis von Bund und Kommunen gehört nach der Arbeitsmarktreform nun die Bildungspolitik. Sie kann nur lokal zum Erfolg geführt werden, obwohl die zentralen Steuerungselemente auf Landes- und Bundesebene entschieden werden. Lern- und Bildungslandschaften (Luthe 2009, S. 613 ff.; Hummel 2010b; Bleckmann 2009) beschreiben die systematische Verknüpfung von Erziehung, Betreuung, Bildung, Lernen und Qualifizierung aller Generationen im lokalen Raum. Zur Entstehung solcher Bildungslandschaften ist eine Wechselwirkung mit der Stadtentwicklung und Planung sowie unternehmerischem Handeln im Nahbereich notwendig. »Eine [bildungspolitische, Anm. K. H.] Kompensation [der Defizite bestimmter Elternhäuser, Anm. K. H.]), die lediglich in den Bildungsinstitutionen selbst ansetzt, kann notwendig nur eine begrenzte Reichweite haben. « (Grundmann et al. 2006, S. 250)

Falsch wäre es nun, die Schule übergriffig etwa so auszustatten, dass sie selbst in 
Elternhäusern interviewt oder Ganztagsschulen zu Umerziehungseinrichtungen machte. Falsch wäre dies vor allem deshalb, weil die Schule selbst selektiv wirkt und zuerst diese Hausaufgabe erledigen muss. Und falsch wäre es auch deshalb, weil es diesen Elternhäusern im sozialen Raum an Optionen in Bezug auf Netzwerke, Nachbarschaft(en) fehlt.

Genau diese Nachbarschaftselemente werden zu zentralen Erfolgsfaktoren der gesamten aktuellen Bildungsreformdebatte. Schulreform braucht sozialräumliche Netzwerk- und Familienhilfearbeit. Wer dieser »[k]ognitiven Kompetenz « (ebd.), präventiver Eltern-Kind-Verstehensarbeit (vgl. Liebenwein 2008, S. 290) und Handlungsbefähigung (vgl. Otto/Ziegler 2010) nicht den Raum in zeitgemäßer hilfreicher Nachbarschaft zugesteht und ihn gestaltet, versäumt die Bedeutung der Nachbarschaft für die gesellschaftlich gerechte Bewältigung der Zukunft. Nachbarschaftsarbeit bedeutet im Konzept der Lernlandschaften, das Gewicht elterlicher, zivilgesellschaftlicher Akteure zu erhöhen, den Stellenwert informellen und nonformalen Lernens zu verbessern und die Netzwerkchancen von Kindern und Eltern zu erhöhen.

Je mehr Vorgänge von der Ausbildung bis zur Rente (mit unterschiedlichsten Tätigkeitsformen und Versicherungswegen), vom Sozialverhalten bis zum Gesundheitsverhalten und Lebensstil zur Disposition stehen, desto wichtiger sind die Kriterien der Entscheidung. Die Betroffenen müssen, je mehr sie "riskant « auswählen, den Instanzen vertrauen, auf die sie sich stützen.

Die Kehrseite der aktiven lebensweltlichen Teilhabe der Bürger ist die Entscheidungsnot, die Lebenschancen überhaupt im Widerstreit aller Optionen und Alternativen nutzen zu können: "Die Stadtpolitik hat ihre Eingriffe bis auf die Intimbereiche der Gesellschaft ausgeweitet: Informelle soziale Netze, urbane Milieus, Lebens-, Denk- und Verhaltensweisen [...]« (Siebel 2010, S. 25 ff.). Walter Siebel nennt dies einen »Prozess der Vergesellschaftung « (ebd.). Der Prozess der Vergesellschaftung korrespondiert damit, dass vermeintlich objektive Kriterien dessen, was gut für den Patienten, das Kind oder den Klienten ist, nicht mehr einem eindeutigen gesellschaftlichen Konsens unterliegen, sondern sich auf "verschiedene Wahrheiten « stützen.

Wenn das objektive »Kindeswohl « und der subjektive »Kindeswillen" in Ein- klang gebracht werden müssen, bleibt nur ein Modell des Aushandelns (vgl. Oelkers/Otto/Ziegler 2010, S. 85 ff.) zwischen Wissenschaft, Ämtern und Betroffenen - und das auf allen Ebenen des Alltags und erst recht ständig dort, wo die öffentliche Hand in das private Leben der Anderen (in der Regel transferabhängiger, bildungsferner Menschen) eingreifen muss.

Es ist zur gängigen Forderung geworden, dass an dieser Schnittstelle das Fördern und Fordern greifen soll, dass also die Betroffenen zum Mitagieren auf dieser Ebene zu befähigen und zu qualifizieren sind. Letztlich ist es die Befähigung, »trotz alledem « produktiv, prosozial zu handeln. »Die Ambivalenzen [...] widerlegen nicht die Notwendigkeit solcher Politik. «(Siebel 2010, S. 36)

Allzu leicht wird übersehen, dass dieses Postulat beinhalten muss, dass die Betroffenen ihre Wahl auch anders fällen oder verlangen können, dass die andere Seite (öffentliche Hand, Institutionen und Träger) sich ebenfalls ändern, öffnen und qualifizieren soll. Der Wandel der Institutionen steht viel zu wenig im Mittelpunkt der Modernisierungsdebatte. Oftmals müssen die Institutionen zuerst ihre Daten, Statistiken, Anweisungen, Abrechnungen »kompatibler « machen!

Diese Entwicklung neuer Nachbarschaftlichkeit ist nicht nur eine andere Art, das gleiche Geschäft zu betreiben. Es verändert die Spielregeln des Zusammenwirkens. Es ändert nicht nur Produktionsprozesse, sondern auch Produkte und Ergebnisse. Es verschärft den Druck, den Anforderungen von Institutionen wie Kita, Schule oder Behörde standzuhalten und die eigene Persönlichkeit, kognitive und kreative Fähigkeiten zu entfalten (vgl. Grundmann 2006, S. 197) und das Nichtwissen auszunutzen, um das Entscheiden zu lernen (vgl. Dabrock 2010, S. 17 ff.).

Schon allein deshalb ist nichts gewonnen, wenn aus den jetzigen Schulen nur eine über den ganzen Tag verteilte (gleiche) Schule wird oder aus der bisherigen politischen Teilhabe in Form von Stadträten, Beiräten und Personalräten nur noch mehr Räte werden. Das, was in solchen Räten verteilt, entschieden, angeboten oder organisiert wird, bedarf selbst integrierter Überprüfung. Erziehung, Wissen, Bildung und Jugendförderung müssen ebenso zusammen gedacht werden, wie es »Paketlösungen « von Woh- nen, Arbeit, Integration etc. geben muss. In vielen Fällen herrschen bei Unternehmen, Verbänden und Institutionen völlig irrelevante Marktbilder konkurrierender Anbieter, die eigentlich zusammenarbeiten müssten.

\section{Integrierte Nachbarschaft als Steuerungsfrage der Kommune}

Von außen betrachtet ist schnell Kritik zu üben, sozialräumliche Arbeit erschöpfe sich in Beziehungsarbeit (vgl. Heiner bei Lüders 2008) oder in Zuständigkeiten und reduziere den Auftrag, zu qualifizieren und zu bilden, leichtfertig auf die Schaffung von Sozialkapital (vgl. Andresen/Otto/Ziegler 2010, S. 165 ff.). In der Tat ist ein Arbeitsansatz sehr schwer integriert und balanciert zu halten, der den Eigenwert und emanzipativen Gehalt von Bildung gleichermaßen mitsichert und Beziehungsarbeit erfolgreich in Strukturen der » urban governance «, der zielorientierten Zusammenarbeit aller Akteure einbettet. Christian Lüders (2008, S. 37) formuliert die hohen Ansprüche und zeigt selbst im Schlusssatz die Konsequenzen: »Deutlich erfolgreicher sind diese Ansätze - und das belegen alle Erfahrungen aus dem europäischen Ausland - allerdings in einem politischen Umfeld, das auf die verbindliche Einbindung aller zu beteiligenden Akteure vor Ort setzt und als die zentralen Handlungsmodi Verhandlung und Koordination auf der Basis gemeinsamer Problemdefinitionen anerkennt. Damit einher geht allerdings die Ausweitung der Sphäre des Politischen in die Stadtgesellschaft mit all den damit verbundenen Implikationen in Bezug auf Beteiligung und Teilhabe. Mit anderen Worten: Für die Verwaltung wird das Leben nicht einfacher.«

Das Leben wird für die Verwaltung nicht einfacher, ebenso wie für den Bürger und für beteiligte Unternehmen. Noch sind »corporate governance « und »corporate social responsibility « nicht die Regel (vgl. Hummel 2010c), und wir tun gut daran, dass für alle Sektoren und Ebenen gilt, was David G. Rand et al. (2009) schlicht die Notwendigkeit nennt, durch positive Interaktionen öffentliche Kooperationen zu befördern. Mit Sanktionen, Misstrauen, Belehrungen von oben und Gesetzen ist für diesen Qualitätssprung von Kooperation und "governance « nichts gewonnen. Sogar die klassischen Prinzipien der Gewal- 
tenteilung und Herrschaftskontrolle sind allein nur begrenzt geeignet, neue »urban governance «zu stützen.

Es stellt eine der größten Herausforderungen sozialräumlicher Arbeit dar, ein politisches Steuerungsgeflecht zu begründen, das den integrierten Ansatz sichert und dennoch zu bewältigen und positiv bleibt. Es reicht in keinem Fall der formale Akt von Zielvereinbarungen und Haushaltskontrollen - ebenso wenig genügt die qualifizierte Selbststeuerung. Es wird Formen der geregelten Teilhabe der Betroffenen auch im Sinne kooperativer Mitverantwortung brauchen, wie Gre- mien, die nicht nur innerfachlich besetzt sind und Zukunftsfragen stellen können. Das Geflecht an Steuerung hat eine funktionale Dimension. Es verknüpft die praktische mit der legitimatorischen Arbeit (Entscheiderstrukturen) und Formen des Wertediskurses (Einstellungsmuster, Milieus) und braucht dafür Institutionen und Rollen. Offensichtlich fehlen uns für die Sprachbefähigung bestimmter Gruppen der Stadt (auch neuer Milieus) oder der Vermittlung nicht mehr selbstverständlicher Zukunftsinvestitionen »Übersetzer " zwischen Feld und Verwaltung (vgl. Stein 2010, S. 68).
Statt jedes Mal neue Stabsstellen und Projekte zu begründen, sollte es »lernende Strukturen « geben. Allerdings muss das kommunal gewollt und angeregt werden. Es wird auch erfordern, dass alle Zwischenraumangebote vom Senioren- bis zum Mehrgenerationenbüro enger zusammenrücken müssen. Ihre Stärke wird nicht die kartellähnliche Lobbyarbeit sein, sondern die vielfältige Stimme der engagierten Bürger selbst. Ihr Gehör zu verschaffen, verhindert den Rückfall in Bürokratie, Staatssteuerung und soziale Kontrolle.

\section{Literatur}

ANDRESEN, S.; OTTO, H.-U.; ZIEGLER, H.: Bildung as Human Development: An educational view on the Capabilities Approach, in: OTTO, H.-U.; ZIEGLER, H. (Hg.): Capabilities - Handlungsbefähigung und Verwirklichungschancen in der Erziehungswissenschaft. 2. Aufl. Opladen 2010, S. 195-197. BLECKMANN, P.; DURDEL, A. (Hg.): Lokale Bildungslandschaften: Perspektiven für Ganztagsschulen und Kommunen. Wiesbaden 2009.

DABROCK, P.: Befähigungsgerechtigkeit als Ermöglichung gesellschaftlicher Inklusion, in: OTTO, H.-U.; ZIEGLER, H. (Hg.): Capabilities - Handlungsbefähigung und Verwirklichungschancen in der Erziehungswissenschaft. 2. Aufl. Opladen 2010, S. 17-53.

DEPARTMENT FOR COMMUNITIES AND LOCAL GOVERNMENT: What works in community cohesion. London 2007; deutsche Kurzfassung: HUMMEL, K. (2009): Erfolgskriterien für den Zusammenhalt unserer Städte, in: Forum Wohnen und Stadtentwicklung Heft 3/2009, vhw e. V., S. 130-134.

DÖRNER, A.; VOGT, L.: Das Geflecht aktiver Bürger. »Kohlen « - eine Stadtstudie zur Zivilgesellschaft im Ruhrgebiet. Wiesbaden 2008.

EUROPÄISCHE UNION: Leipzig Charta zur nachhaltigen europäischen Stadt, Internet: http://www.eu2007.de/de/ News/download_docs/Mai/0524-AN/075DokumentLeipzigCharta.pdf, Stand: 24.05.2007, Abruf: 20.05.2010.

FREY, O. (2009): Die amalgame Stadt: Orte. Netze. Milieus. Wiesbaden.

GRUNDMANN, M. [et al.] (2006): Handlungsbefähigung und Milieu. Zur Analyse milieuspezifischer Alltagspraktiken und ihrer Ungleichheitsrelevanz. Münster/New York.

GRUNOW, D.: Kommunalisierung der Sozialpolitik: Theoretische Grundlagen und Herausforderungen, in: DAHME, H. J.; WOHLFAHRT, N. (Hg.): Regiert das Lokale das Soziale? Die Kommunalisierung und Dezentralisierung sozialer Dienste als sozialpolitische Reformstrategie. Baltmannsweiler 2010, S. 10-25.

HUMMEL, K.: Stadtentwicklung und Perspektiven der Bürgergesellschaft. Unveröffentlichtes Manuskript. Berlin 2010 [2010a].
HUMMEL, K.: Auf dem Weg zu Lernlandschaften - Stichworte zu einem politischen Topthema. Unveröffentlichtes Manuskript. Berlin 2010 [2010b].

HUMMEL, K.: Gesellschaftliche Verantwortung in der Wohnungswirtschaft: Eine Positionsbestimmung aus Sicht des vhw - Bundesverband für Wohnen und Stadtentwicklung e. V., Arbeitspapier. Berlin 2010 [2010c].

LIEBENWEIN, S.: Erziehung und soziale Milieus. Elterliche Erziehungsstile in milieuspezifischer Differenzierung. Wiesbaden 2008.

LUTHE, E.-W.: Kommunale Bildungslandschaften - Bildung als Integrationswert der örtlichen Gemeinschaft, in: Der Landkreis (2009), S. 613-619.

LÜDERS, C.: Sozialraumdiskussion quo vadis? Marginalien $\mathrm{zu}$ den Tagungsthesen in: Tagungsdokumentation Sozialraumorientierung. Segregation, Marginalisierung und Integration in urbanen Räumen. München, 15.02.2008. Internet: http://www.dji.de/bibs/64_Tag_Sozialraum.pdf, S. 34-38, Abrufdatum: 21.05.2010.

OELKERS, N.; OTTO, H.-U.; ZIEGLER, H.: Handlungsbefähigung und Wohlergehen - Der Capabilities-Ansatz als alternatives Fundament der Bildungs- und Wohlfahrtsforschung, in: OTTO, H.-U.; ZIEGLER, H. (Hg.): Capabilities Handlungsbefähigung und Verwirklichungschancen in der Erziehungswissenschaft. 2. Aufl. Opladen 2010, S. 85-89.

OTTO, H.-U.; ZIEGLER, H. (Hg.): Capabilities - Handlungsbefähigung und Verwirklichungschancen in der Erziehungswissenschaft. 2. Aufl. Opladen 2010.

RAND, D. G. [et al.]: Positive Interactions Promote Public Cooperation, in: Science, vol. 325 (2009), S. 1272-1275.

SIEBEL, W.: Planende Verwaltung und zivile Gesellschaft, in: STRACHWITZ, R. (Hg.): Stadtentwicklung, Zivilgesellschaft und bürgerschaftliches Engagement. Stuttgart 2010, S. 25-38.

STEIN, M.: Zivilgesellschaft und Stadtentwicklung: Diskussionspapier zu einem Expertengespräch der Initiative „Stadt als Campus“, in: Planerin - Fachzeitschrift für Stadt-, Regional- und Landesplanung, 2 (2010), S. 65-68.

ZEUNER, N.: Soziale Stadtentwicklung durch Urban Governance, in: Neue Gesellschaft/Frankfurter Hefte, (2009), S. 37-39. 